\title{
ON THE FACTOR ALPHA IN PEYRE'S CONSTANT
}

\author{
ULRICH DERENTHAL, ANDREAS-STEPHAN ELSENHANS, AND JÖRG JAHNEL
}

Abstract. For an arbitrary del Pezzo surface $S$, we compute $\alpha(S)$, which is the volume of a certain polytope in the dual of the effective cone of $S$, using magma and polymake. The constant $\alpha(S)$ appears in Peyre's conjecture for the asymptotic formula for the number of rational points of bounded height on $S$ over number fields.

\section{INTRODUCTION}

Let $S$ be a smooth del Pezzo surface defined over a number field $k$; for simplicity, we assume in this introduction that its degree is $\leq 7$. If $S$ contains infinitely many $k$-rational points, Manin's conjecture [11 makes a precise prediction of the number of $k$-rational points of bounded height on $S$ : Let $U$ be the complement of the lines (more precisely, (-1)-curves; see Section 2 for details) on $S$, and let $H$ be an anticanonical height function on the set $S(k)$ of $k$-rational points. Then the number

$$
N_{U, H}(B)=\#\{\mathbf{x} \in U(k) \mid H(\mathbf{x}) \leq B\}
$$

of $k$-rational points of height at most $B$ outside the lines is expected to grow asymptotically as $c_{S, H} B(\log B)^{\varrho(S)-1}$ for $B \rightarrow \infty$, where $\varrho(S)$ is the rank of the Picard group $\operatorname{Pic}(S)$ over $k$, and $c_{S, H}>0$ is a constant whose value was predicted by Peyre [17 to be

$$
c_{S, H}=\alpha(S) \beta(S) \omega(S, H) .
$$

See 4 for an overview of this conjecture for del Pezzo surfaces, and, e.g., 10] for experimental results. Here, $\omega(S, H)$ is essentially a product of local densities that shows up similarly, for example, in applications of the Hardy-Littlewood circle method, $\beta(S)$ is defined as $\# H^{1}\left(\operatorname{Gal}(\bar{k} / k), \operatorname{Pic}\left(S_{\bar{k}}\right)\right)$, and $\alpha(S)$ is the volume of a certain polytope in $\operatorname{Pic}(S)_{\mathbb{R}}=\operatorname{Pic}(S) \otimes_{\mathbb{Z}} \mathbb{R} \cong \mathbb{R}^{\varrho(S)}$.

In this note, we are interested in $\alpha(S)$. For any smooth del Pezzo surface $S$, it can be defined as follows; see also [17, Définition 2.4] and [1, Definition 2.4.6]

Definition 1.1. Let $\Lambda_{\text {eff }}(S)$ be the effective cone of $S$ in $\operatorname{Pic}(S)_{\mathbb{R}}$, let $\Lambda_{\text {eff }}^{\vee}(S)$ be its dual cone with respect to the intersection form, and let $\left(-K_{S}\right) \in \operatorname{Pic}(S)$ be the anticanonical class of $S$. Then

$$
\alpha(S)=\varrho(S) \cdot \operatorname{vol}\left\{x \in \Lambda_{\text {eff }}^{\vee}(S) \mid\left\langle x,-K_{S}\right\rangle \leq 1\right\},
$$

Received by the editor February 28, 2012.

2010 Mathematics Subject Classification. Primary 14J26; Secondary 51M20, 14G05.

Key words and phrases. Peyre's constant, del Pezzo surface, polyhedron, volume, polymake.

The first author was partly supported by Deutsche Forschungsgemeinschaft (DFG) grant DE 1646/2-1, SNF grant 200021_124737/1, and by the Center for Advanced Studies of LMU München.

The second author was supported in part by the Deutsche Forschungsgemeinschaft (DFG) through a funded research project. 
where the volume on $\operatorname{Pic}(S)_{\mathbb{R}}$ is normalized such that the lattice $\operatorname{Pic}(S)$ has covolume 1 .

Over an algebraic closure $\bar{k}$ of $k$, the effective cone $\Lambda_{\text {eff }}\left(S_{\bar{k}}\right)$ is generated by the lines on $S_{\bar{k}}$. We will see that $\alpha(S)$ depends only on the degree $d$ of $S$ and on the combinatorial structure of the action of $\operatorname{Gal}(\bar{k} / k)$ on the lines on $S_{\bar{k}}$ via a subgroup $W(S)$ of their finite symmetry group $W$, which is a Weyl group.

For smooth split del Pezzo surfaces $(\# W(S)=1$, i.e., when each line is defined over $k$ ), a formula for $\alpha(S)$ was found in [7, Theorem 4]. For smooth non-split del Pezzo surfaces of degree $\geq 5$, the values of $\alpha(S)$ were determined in [8, Section 7B].

Remark 1.2. For a del Pezzo surfaces $S^{\prime}$ with ADE singularities, Manin's conjecture can be formulated similarly. In this case, the expected constants must be computed on the minimal desingularization $\widetilde{S}^{\prime}$ of $S^{\prime}$. We have $\alpha\left(\widetilde{S}^{\prime}\right)=\frac{\alpha(S)}{\# W^{\prime}}$, where $S$ is a smooth del Pezzo surface of the same degree as $S^{\prime}$ with a corresponding action of $\operatorname{Gal}(\bar{k} / k)$ on its lines over $\bar{k}$ and $W^{\prime}$ is a Weyl group associated to the singularities of $S^{\prime}$. See [8, Theorem 1.3, Corollary 7.5] for details.

It remains to determine $\alpha(S)$ for smooth non-split del Pezzo surfaces of degree $d \leq 4$. By Remark 1.2 this is also relevant for the singular case. Unfortunately, it seems that this cannot be done without certain case-by-case considerations. We will give a general algorithm and then apply it to the cases $d=1, \ldots, 4$.

By [8, Section 7A], we are reduced to finitely many cases corresponding to the conjugacy classes of subgroups of the Weyl groups $W$. For example, when $d=1$, the number of cases is 62092 ; for cubic surfaces, there are 350 cases.

Theorem 2.2 is our key result that allows us to reduce the number of cases to an order of magnitude that could in principle be treated by hand without too much effort: If $S$ and $S^{\prime}$ have the same degree, Picard groups of the same rank and if $W(S)$ is contained in a conjugate of $W\left(S^{\prime}\right)$, then $\alpha(S)=\alpha\left(S^{\prime}\right)$. Using this, only 14 cases are left for $d=4$, only 17 for $d=3$, only 32 for $d=2$, and 41 for $d=1$.

Nevertheless, we choose to treat these cases not by hand, but with the help of the software polymake 13 that allows us to compute volumes of polytopes. The subgroups of $W\left(R_{d}\right)$ can be obtained using gap [12] or magma [2]. Our results are summarized in Tables 2 , 3 and 4.

At least for $d \geq 3$, it is possible to extract the value of $\alpha(S)$ for a concretely given del Pezzo surface from these tables. See Remark 4.6 for a detailed discussion.

\section{Del Pezzo surfaces}

We recall some facts on the structure of del Pezzo surfaces. See 16 for more details. A (smooth / ordinary) del Pezzo surface $S$ over a field $k$ is a smooth projective variety of dimension 2 defined over $k$ whose anticanonical class $\left(-K_{S}\right)$ is ample. Its degree $d$ is the self-intersection number $\left(-K_{S},-K_{S}\right)$ of the anticanonical class. Over $\bar{k}$, it is isomorphic to $\mathbb{P}^{2}$ (of degree 9 ), $\mathbb{P}^{1} \times \mathbb{P}^{1}$ (of degree 8 ) or the blow-up of $\mathbb{P}^{2}$ in $r$ points in general position (with $r \in\{1, \ldots, 8\}$, of degree $d=9-r$ ). Its geometric Picard group $\operatorname{Pic}\left(S_{\bar{k}}\right)$ is free of rank $10-d$.

A $(-1)$-curve is a curve $E$ on $S_{\bar{k}}$ such that its class $[E] \in \operatorname{Pic}\left(S_{\bar{k}}\right)$ satisfies $\left(-K_{S},[E]\right)=1$ and $([E],[E])=-1$. A $(-2)$-class is an element $L$ of $\operatorname{Pic}\left(S_{\bar{k}}\right)$ with $\left(-K_{S}, L\right)=0$ and $(L, L)=-2$.

From here, we restrict to the case $d \leq 7$. Then $\operatorname{Pic}\left(S_{\bar{k}}\right)$ and its intersection form depend only on the degree $d$ of $S$ (cf. [8, Section 3]). Consequently, the same 
TABLE 1. The root systems associated to del Pezzo surfaces of degree $\leq 4$

\begin{tabular}{|c||c|c|c|c|}
\hline$d$ & 4 & 3 & 2 & 1 \\
\hline \hline$R_{d}$ & $\mathbf{D}_{5}$ & $\mathbf{E}_{6}$ & $\mathbf{E}_{7}$ & $\mathbf{E}_{8}$ \\
$\# W\left(R_{d}\right)$ & 1920 & 51840 & 2903040 & 696729600 \\
\hline$w_{d}$ & 1328 & 1161 & - & - \\
$c_{d}$ & 197 & 350 & 8074 & 62092 \\
$c_{d}^{\prime}$ & 38 & 91 & 1071 & 13975 \\
$c_{d}^{\prime \prime}$ & 14 & 17 & 32 & 41 \\
\hline$N_{d}$ & 16 & 27 & 56 & 240 \\
$N_{d, 0}$ & 10 & 16 & 27 & 56 \\
$N_{d, 1}$ & 5 & 10 & 27 & 126 \\
$N_{d, 2}$ & 0 & 0 & 1 & 56 \\
$N_{d, 3}$ & 0 & 0 & 0 & 1 \\
\hline
\end{tabular}

holds for the $(-2)$-classes (which form a root system $R_{d}$ whose type can be found in Table 1 for $d \leq 4$ ) and the classes of the $(-1)$-curves (whose number is denoted by $N_{d}$ ) with their pairwise intersection numbers.

The geometric Picard group $\operatorname{Pic}\left(S_{\bar{k}}\right)$ is generated by the classes of the $(-1)$ curves. The symmetry group of $\operatorname{Pic}\left(S_{\bar{k}}\right)$ respecting the intersection form is the Weyl group $W\left(R_{d}\right)$ associated to the root system $R_{d}$ of the $(-2)$-classes in $\operatorname{Pic}\left(S_{\bar{k}}\right)$. Via its action on the set of $(-1)$-curves, $W\left(R_{d}\right)$ can be regarded as a subgroup of the symmetric group $\mathrm{S}_{N_{d}}$. Over $\bar{k}$, the effective cone $\Lambda_{\text {eff }}\left(S_{\bar{k}}\right) \subset \operatorname{Pic}\left(S_{\bar{k}}\right)_{\mathbb{R}}$ is generated by the $(-1)$-curves (see [8, Proposition 3.9], for example). All of this depends only on the degree $d \leq 7$.

Remark 2.1. For $d \leq 5$, the group $W\left(R_{d}\right)$ acts transitively on the sets of pairs of $(-1)$-curves with fixed intersection number (in the set $\{-1, \ldots, 3\}$ ). This gives a way to recover $\operatorname{Pic}\left(S_{\bar{k}}\right)$ with the intersection form and the classes of the $(-1)$-curves if $W\left(R_{d}\right)$ is given as a subgroup of $\mathrm{S}_{N_{d}}$. Namely, any (-1)-curve $E$ has intersection number $i$ with precisely $N_{d, i}$ other $(-1)$-curves, as listed in Table1, Therefore, the pairs of $(-1)$-curves with intersection number $i$ form an orbit with precisely $\frac{N_{d} \cdot N_{d, i}}{2}$ elements under the action of $W\left(R_{d}\right)$. This gives an $N_{d} \times N_{d}$ intersection matrix $M_{d}$ of rank $10-d$, with $\operatorname{Pic}\left(S_{\bar{k}}\right) \cong \mathbb{Z}^{N_{d}} / \operatorname{ker} M_{d}$.

For $d \in\{1,2\}$, we have $N_{d, 0}=N_{d, 3-d}$, giving two orbits of the same size. This results in two candidates for $M_{d}$, where the correct one can be identified by the expected rank.

However, $\operatorname{Pic}(S)$ and $\alpha(S)$ depend fundamentally on the structure of $S$ over $k$, via the natural action of the Galois group $\operatorname{Gal}(\bar{k} / k)$ on $\operatorname{Pic}\left(S_{\bar{k}}\right)$. Since this action respects the intersection pairing, it factors via a subgroup $W(S)$ of $W\left(R_{d}\right)$. The group $W(S)$ acts on the set of $(-1)$-curves, breaking it into $n$ orbits $\left\{E_{i, 1}, \ldots, E_{i, k_{i}}\right\}$ of size $k_{i}$, for $i=1, \ldots, n$.

Since $\alpha(S)$ is irrelevant if $S(k)=\emptyset$, we assume once and for all that $S$ has a $k$-rational point. Then $\operatorname{Pic}(S)=\operatorname{Pic}\left(S_{\bar{k}}\right)^{\operatorname{Gal}(\bar{k} / k)}$ by [6, Theorem 2.1.2, Claim (iii)]. Consequently, $\Lambda_{\text {eff }}(S)$ is generated by the classes of $E_{i, 1}+\cdots+E_{i, k_{i}}$, for $i=1, \ldots, n$.

Therefore, $\alpha(S)$ depends only on the conjugacy class of $W(S)$ in $W\left(R_{d}\right)$, which reduced the computation of all cases of $\alpha(S)$ to a finite problem. We denote the 
number of conjugacy classes of subgroups of $W\left(R_{d}\right)$ by $c_{d}$; see Table 1. More precisely, it depends only on the orbit structure of the action of $W(S)$ on the set of $(-1)$-curves, up to conjugacy, reducing the problem to $c_{d}^{\prime}$ classes in degree $d$.

For $d \leq 6$, the sum of the classes of the $(-1)$-curves in $\operatorname{Pic}\left(S_{\bar{k}}\right)$ is $\frac{N_{d}}{d} \cdot\left(-K_{S}\right)$. Indeed, this sum is invariant under $W\left(R_{d}\right)$, so it is a scalar multiple of $\left(-K_{S}\right)$. The value of this scalar is determined from the fact that $\left(-K_{S},-K_{S}\right)=d$ and $\left([E],-K_{S}\right)=1$ for each $(-1)$-curve $E$.

Theorem 2.2. Let $S_{1}, S_{2}$ be del Pezzo surfaces of degree $d \leq 7$ over a field $k$ such that $S_{1}(k), S_{2}(k) \neq \emptyset$. For the corresponding subgroups $W\left(S_{1}\right), W\left(S_{2}\right)$ of $W\left(R_{d}\right)$, suppose that $W\left(S_{1}\right) \subseteq g W\left(S_{2}\right) g^{-1}$ for some $g \in W\left(R_{d}\right)$. Further, assume $\operatorname{rk} \operatorname{Pic}\left(S_{1}\right)=\operatorname{rkPic}\left(S_{2}\right)$. Then $\alpha\left(S_{1}\right)=\alpha\left(S_{2}\right)$.

Proof. As $\operatorname{Pic}\left(S_{i}\right)=\operatorname{Pic}\left(\left(S_{i}\right)_{\bar{k}}\right)^{W\left(S_{i}\right)}$, we see that $\operatorname{Pic}\left(S_{i}\right)$ are maximal sublattices. On the other hand, up to isometry, $\operatorname{Pic}\left(S_{1}\right) \supseteq \operatorname{Pic}\left(S_{2}\right)$. Thus, the equality of the ranks implies that $\operatorname{Pic}\left(S_{1}\right)=\operatorname{Pic}\left(S_{2}\right)$. Further, every $W\left(S_{2}\right)$-orbit of $(-1)$-curves breaks into one or several $W\left(S_{1}\right)$-orbits. If $\left\{E_{1,1}, \ldots, E_{k, l_{k}}\right\}$ is a $W\left(S_{2}\right)$-orbit that breaks into the $W\left(S_{1}\right)$-orbits $\left\{E_{i, 1}, \ldots, E_{i, l_{i}}\right\}$, for $i=1, \ldots, k$, then $\left[E_{i, 1}\right]+\cdots+\left[E_{i, l_{i}}\right] \in \operatorname{Pic}\left(S_{1}\right)=\operatorname{Pic}\left(S_{2}\right)$ is $W\left(S_{2}\right)$-invariant and therefore independent of $i$. In particular,

$$
\left[E_{i, 1}\right]+\cdots+\left[E_{i, l_{i}}\right]=\frac{1}{k}\left(\left[E_{1,1}\right]+\cdots+\left[E_{k, l_{k}}\right]\right) \in \operatorname{Pic}\left(S_{1}\right)_{\mathbb{R}} .
$$

Hence, $\Lambda_{\text {eff }}\left(S_{1}\right)=\Lambda_{\text {eff }}\left(S_{2}\right)$.

2.3. Let $S$ be of degree $d$. The stabilizer $G$ of $\operatorname{Pic}(S)$ for the action of $W\left(R_{d}\right)$ on $\operatorname{Pic}\left(S_{\bar{k}}\right)$ is the unique subgroup of $W\left(R_{d}\right)$ containing $W(S)$ that is maximal with the property that $\varrho(S)=\operatorname{rk}\left(\operatorname{Pic}\left(S_{\bar{k}}\right)^{G}\right)$. We call each such $G$ a $\varrho$-maximal subgroup of $W\left(R_{d}\right)$. Let $c_{d}^{\prime \prime}$ be the number of conjugacy classes of $\varrho$-maximal subgroups of $W\left(R_{d}\right)$ (cf. Table 1). Theorem 2.2 shows that it is enough to compute $\alpha(S)$ in the corresponding $c_{d}^{\prime \prime}$ cases.

\section{The ALGORITHM}

Algorithm 3.1 $(\alpha(S)$ for del Pezzo surfaces $S$ of degree $d \leq 5)$.

I) Realize in gap or magma the group $W\left(R_{d}\right)$ as a subgroup of the symmetric group $\mathrm{S}_{N_{d}}$, identifying the set of all lines with $\left\{1, \ldots, N_{d}\right\}$.

II) Determine the $N_{d} \times N_{d}$ intersection matrix $M_{d}$, using Remark 2.1,

III) The geometric Picard group of rank $10-d$ is now isomorphic to $\mathbb{Z}^{N_{d}} / \operatorname{ker} M_{d}$. Select $(-1)$-curves $E_{1}, \ldots, E_{9-d}$ that are pairwise skew and a $(-1)$-curve $E_{10-d}$ that is distinct from the previous ones. Ensure that the corresponding $(10-d) \times(10-d)$ intersection matrix is of determinant \pm 1 . Then $\left\{\left[E_{1}\right], \ldots,\left[E_{10-d}\right]\right\}$ forms a basis of the geometric Picard group. Express every line in this basis. This yields a map $p:\left\{1, \ldots, N_{d}\right\} \rightarrow \mathbb{Z}^{10-d}$, represented by a matrix.

IV) Calculate a set $U_{d}$ of representatives of the $c_{d}$ conjugacy classes of subgroups in $W\left(R_{d}\right)$, together with the number of subgroups in each conjugacy class.

V) For each subgroup $G \in U_{d}$, compute the $n_{G}$ orbits of $G$ acting on $\left\{1, \ldots, N_{d}\right\}$. Compute the rank $\varrho_{G}$ of $\operatorname{Pic}\left(S_{\bar{k}}\right)^{G}$, which is the rank of the $n_{G} \times n_{G}$ matrix whose 
entry $(i, j)$ is the intersection number (computed using $M_{d}$ ) of the sums of elements in the $i$-th and $j$-th orbit.

VI) Repeat the following until $U_{d}$ is empty, producing a set $M_{d}$ (initially empty, at the end of order $\left.c_{d}^{\prime \prime}\right)$ of subgroups of $W\left(R_{d}\right)$ such that for any subgroup $H$ there is a unique $G \in M_{d}$ with $\varrho_{G}=\varrho_{H}$ containing a conjugate of $H$.

i) Choose a subgroup $G \in U_{d}$ of maximal order and add it to $M_{d}$.

ii) Remove all $H$ from $U_{d}$ with $\varrho_{H}=\varrho_{G}$ contained in a conjugate of $G$.

VII) For each $G \in M_{d}$, compute the corresponding value of $\alpha(S)$ as follows:

i) For each orbit $\left\{l_{i, 1}, \ldots, l_{i, k_{i}}\right\}$ of $G$ acting on $\left\{1, \ldots, N_{d}\right\}$ (with $i=1, \ldots, n_{G}$ ), calculate the vector $v_{i}=p\left(l_{i, 1}\right)+\cdots+p\left(l_{i, k_{i}}\right) \in \mathbb{Z}^{10-d}$. This yields a list $v_{1}, \ldots, v_{n_{G}}$ of vectors in $\mathbb{Z}^{10-d}$.

ii) Determine a basis of the free $\mathbb{Z}$-module $\mathbb{Z}^{10-d} \cap\left(\mathbb{Q} v_{1}+\cdots+\mathbb{Q} v_{n_{G}}\right) \cong \mathbb{Z}^{\varrho}$, which is isomorphic to the Picard group. Express the vectors $v_{1}, \ldots, v_{n_{G}}$ in this basis and print the list of coefficient vectors $w_{1}, \ldots, w_{n_{G}} \in \mathbb{Z}^{\varrho}$ obtained, together with a marker of the conjugacy class treated, into a file.

iii) Read this file into a polymake script. For each conjugacy class, realize in polymake the polytope in $\mathbb{R}^{\varrho}$, given by $\left\langle x, w_{1}\right\rangle \geq 0, \ldots,\left\langle x, w_{n_{G}}\right\rangle \geq 0$ and $\left\langle x, w_{1}+\cdots+w_{n_{G}}\right\rangle \leq \frac{N_{d}}{d}$. Compute the volume of the polytope, multiply by $\varrho$, and return the result.

Remarks 3.2. a) Steps $\mathrm{V}$ ) and $\mathrm{VI}$ ) use Theorem 2.2 to reduce the number of computations of $\alpha(S)$ significantly from $c_{d}$ to $c_{d}^{\prime \prime}$. This leads to a reasonably short list of results with a natural structure. For $d=1$, this also seems absolutely necessary to keep the running times reasonably low.

To find all $H$ with $\varrho_{H}=\varrho_{G}$ contained in a conjugate of $G$ in $W=W\left(R_{d}\right)$ in magma, one may either compute $\operatorname{Conjugates}(W, G)$ and compare all candidates $H$ with the resulting list for inclusion, or test IsConjugate $(\mathrm{W}, \mathrm{H}, \mathrm{U})$ for all $U$ in Subgroups (G). For $d=1$, depending on the number of conjugates of subgroups of $G$ (which is up to 604800) and candidates $H$ (which is up to 48797), each option might take prohibitively long. It turns out that it is reasonable to take the first approach whenever the number of conjugates, known from the computation of Subgroups (W) in step IV), is less than the number of candidates. With this strategy, we must only deal with up to 1120 conjugates in the first case and up to 1886 candidates in the second case.

b) Computationally, the case of degree $d=1$ is by far the hardest. Using magma V2.17-9 on an Intel Xeon L5640 CPU at $2.27 \mathrm{GHz}$, step IV) took 32 minutes, step $\mathrm{V}$ ) took 68 minutes and step $\mathrm{VI}$ ) took 3 minutes, resulting in 41 conjugacy classes of $\varrho$-maximal subgroups. For $d=2$, steps IV) VI) took a total of 35 seconds giving 32 conjugacy classes, and $d \geq 3$ is negligible.

For $d \geq 3$, one can also use gap to compute the conjugacy classes of subgroups together with a numbering from 1 to $c_{d}$ in step IV) This numbering is reproducible, at least in our version of gap. For $W\left(E_{7}\right)$ and $W\left(E_{8}\right)$, however, gap runs out of memory. The difference comes from the fact that the Cannon/Holt algorithm [5] to determine the maximal subgroups of a given finite group is available in magma. 
c) The running times for the volume computations were as follows, using polymake, version 2.9.9, on an AMD Phenom II X4 955 processor. We describe the polytope by its INEQUALITIES properties. Again, degrees $d \geq 3$ are done in a few seconds.

The 32 cases of del Pezzo surfaces of degree 2 together took 85 seconds of CPU time. Among them, the most complicated case is that of the split del Pezzo surface. Here, according to the definition, one has to compute the volume of a polytope in $\mathbb{R}^{8}$, having 703 vertices. This case alone took 36 seconds; 279 MBytes of memory were being allocated.

The 39 cases of del Pezzo surfaces of degree 1, excluding those of Picard ranks 8 or 9 , together took 140 seconds of CPU time. Here, 300 MBytes of memory were allocated. The rank- 8 case alone, however, took around 37 minutes of CPU time and 3 GBytes of memory. Finally, the split del Pezzo surface of degree one leads to a polytope in $\mathbb{R}^{9}$ with 19441 vertices. Here, polymake fails, as 8 GBytes of working memory turn out to be insufficient. In order to make it work, we incorporated the obvious $\mathrm{S}_{8}$-symmetry of the polytope. Then the volume could be computed within 3.8 seconds, using only 150 MBytes of memory. The same trick works for the rank- 8 case. Here, incorporating the obvious $\mathrm{S}_{6}$-symmetry reduces the running time to 4.8 seconds and the memory usage to 158 MBytes.

d) In the cases when $S$ is isomorphic over $k$ to the blow-up of $\mathbb{P}^{2}$ in some Galoisinvariant set of size $9-d$, one has explicit generators for the Picard group [14, Theorem V.4.9]. Such cases may, in principle, be handled interactively. For example, the following snippet of polymake code computes $\alpha(S)$ for a split cubic surface $S$.

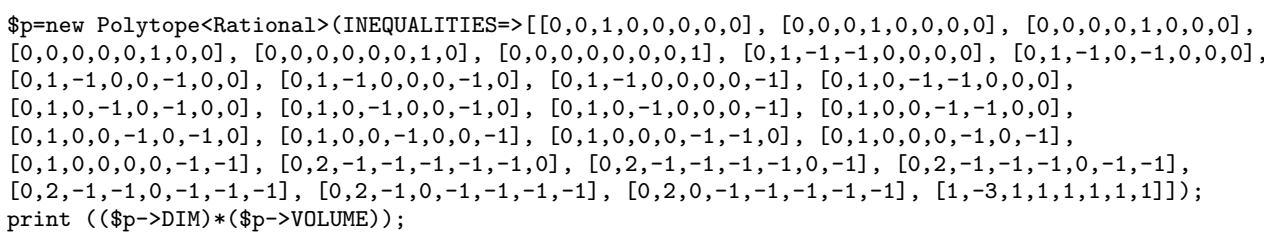

\section{Results}

For $d \geq 5$, the values of $\alpha(S)$ were systematically computed in [8, Section 7B]. For $d=5$, our algorithm recovers the results listed in [8, Table 8]. For $d=4$, the description of the results is relatively straightforward. For the probably most important and interesting case $d=3$ of cubic surfaces, we give more details. For simplicity, we only give an overview of the results for $d=2$ and $d=1$.

Theorem 4.1 (The values of $\alpha(S)$ for quartic del Pezzo surfaces). Let $S$ be a smooth quartic del Pezzo surface over a field $k$ such that $S(k) \neq \emptyset$. Then exactly one of following is true.

I) $\operatorname{rk} \operatorname{Pic}(S)=1$. Then $\alpha(S)=1$.

II) $\operatorname{rk} \operatorname{Pic}(S)=2$. Then there are two cases.

i) $S$ has no k-rational $(-1)$-curve. Then $\alpha(S)=1$.

ii) $S$ is isomorphic to $\mathbb{P}^{2}$, blown up in an orbit of size five. Then $\alpha(S)=\frac{2}{3}$.

III) $\operatorname{rk} \operatorname{Pic}(S)=3$. Then there are two cases. 
i) $S$ has no k-rational $(-1)$-curve. Then $\alpha(S)=\frac{1}{2}$.

ii) $S$ is isomorphic to $\mathbb{P}^{2}$, blown up in a k-rational point and an orbit of size four or in an orbit of size two and an orbit of size three. Then $\alpha(S)=\frac{1}{3}$.

IV) $\operatorname{rk} \operatorname{Pic}(S)=4$. Then there are two cases.

i) $S$ has no k-rational $(-1)$-curve. Then $\alpha(S)=\frac{1}{6}$.

ii) $S$ is isomorphic to $\mathbb{P}^{2}$, blown up in two $k$-rational points and an orbit of size three or in a $k$-rational point and two orbits of size two. Then $\alpha(S)=\frac{1}{9}$.

V) $\operatorname{rk} \operatorname{Pic}(S)=5$. Then $\alpha(S)=\frac{1}{36}$.

VI) $\operatorname{rk} \operatorname{Pic}(S)=6$. Then $\alpha(S)=\frac{1}{180}$.

Proof. Our implementation of Algorithm 3.1 (skipping the reduction step VI) and working with $U_{d}$ instead of $M_{d}$ afterwards) yields a list, associating to each number from 1 to 197 a value of alpha. The result is obtained by giving a geometric interpretation to this list.

Step VI) of Algorithm 3.1 gives 14 conjugacy classes of $\varrho$-maximal subgroups (see 2.3) of $W\left(D_{5}\right)$. In step VII), we discover that this leads to eight distinct values of $\alpha(S)$. Among the 197 conjugacy classes of subgroups and the 38 orbit structures, they are distributed as shown in Table 2 ,

TABle 2. Degree four del Pezzo surfaces, the $14 \varrho$-maximal cases

\begin{tabular}{|c|c||c|c||c|c||c|}
\hline Case & $\alpha$ & $\begin{array}{c}\text { \#conj. } \\
\text { classes }\end{array}$ & $\varrho$-maximal & $\begin{array}{c}\text { \#orbit } \\
\text { structures }\end{array}$ & maximal & $\begin{array}{c}\text { corr. case } \\
\text { cub. surf. }\end{array}$ \\
\hline \hline I & 1 & 98 & $W\left(D_{5}\right)$ & 7 & {$[16]$} & II.i \\
\hline \hline II.i & 1 & 50 & $\mathrm{~S}_{4} \rtimes(\mathbb{Z} / 2 \mathbb{Z})^{3}$ & 12 & {$[8,8]$} & III.i \\
\hline & & 7 & $\mathrm{~S}_{3} \times \mathrm{S}_{2} \times \mathbb{Z} / 2 \mathbb{Z}$ & 1 & {$[2,2,6,6]$} & III.iii \\
\hline & & 11 & $\mathrm{~S}_{4} \times \mathrm{S}_{2}$ & 2 & {$[4,4,8]$} & III.iv \\
\hline II.ii & $\frac{2}{3}$ & 5 & $\mathrm{~S}_{5}$ & 2 & {$[1,5,10]$} & III.v \\
\hline \hline III.i & $\frac{1}{2}$ & 5 & $\mathrm{~S}_{4}$ & 1 & {$[4,4,4,4]$} & IV.i \\
\hline & & 5 & $\mathrm{~S}_{2} \times \mathrm{S}_{2} \times \mathrm{S}_{2}$ & 3 & {$[2,2,2,2,4,4]$} & IV.ii \\
\hline III.ii & $\frac{1}{3}$ & 5 & $\mathrm{~S}_{4}$ & 3 & {$[1,1,4,4,6]$} & IV.i \\
\hline & & 3 & $\mathrm{~S}_{2} \times \mathrm{S}_{3}$ & 1 & {$[1,1,2,3,3,6]$} & IV.iii \\
\hline \hline IV.i & $\frac{1}{6}$ & 2 & $\mathrm{~S}_{2} \times \mathrm{S}_{2}$ & 1 & {$[2,2, \ldots, 2]$} & V.i \\
\hline IV.ii & $\frac{1}{9}$ & 2 & $\mathrm{~S}_{2} \times \mathrm{S}_{2}$ & 2 & {$[1,1,1,1,2,2,2,2,4]$} & V.i \\
\hline & & 2 & $\mathrm{~S}_{3}$ & 1 & {$[1,1,1,1,3,3,3,3]$} & V.ii \\
\hline \hline V & $\frac{1}{36}$ & 1 & $\mathrm{~S}_{2}$ & 1 & {$[1, \ldots, 1,2,2,2,2]$} & VI \\
\hline \hline VI & $\frac{1}{180}$ & 1 & 0 & 1 & {$[1,1, \ldots, 1]$} & VII \\
\hline \hline$\sum$ & & 197 & & 38 & & \\
\hline
\end{tabular}


The rightmost column in this table indicates the type of cubic surface (cf. Theorem 4.2 and Table 3 below) that occurs when blowing up one $k$-rational point. It is a little surprising that the types IV.i and V.i appear twice. The reason for this is as follows.

A quartic del Pezzo surface of type III.i may be constructed by blowing up $\mathbb{P}^{2}$ in two $k$-rational points and an orbit of size four, followed by blowing down the line through the two $k$-rational points. This is a non-blown-up case, but $k$-birationally equivalent to a surface of type III.ii, which may be obtained by blowing up $\mathbb{P}^{2}$ in a $k$-rational point and an orbit of size four. For the cases IV.i and IV.ii, the situation is very similar.

Theorem 4.2 (The values of $\alpha(S)$ for cubic surfaces). Let $S$ be a smooth cubic surface over a field $k$ such that $S(k) \neq \emptyset$. Then exactly one of the following is true.

I) $\operatorname{rk} \operatorname{Pic}(S)=1$. Then $\alpha(S)=1$.

II) $\operatorname{rk} \operatorname{Pic}(S)=2$. Then there are four cases.

i) $S$ has a k-rational line. Then $\alpha(S)=1$.

ii) $S$ is isomorphic to $\mathbb{P}^{2}$, blown up in an orbit of size six. Then $\alpha(S)=\frac{4}{3}$.

iii) $S$ has a Galois-invariant double-six [9]. Over the quadratic extension $l / k$, splitting the double-six, $S_{l}$ is isomorphic to $\mathbb{P}^{2}$, blown up in two orbits of size three. Then $\alpha(S)=2$.

iv) $S$ has a Galois orbit consisting of two skew lines. Then $\alpha(S)=\frac{3}{2}$.

III) $\operatorname{rk} \operatorname{Pic}(S)=3$. Then there are five cases.

i) $S$ has three coplanar $k$-rational lines. Then $\alpha(S)=\frac{1}{2}$.

ii) $S$ is isomorphic to $\mathbb{P}^{2}$, blown up in two orbits of size three. Then $\alpha(S)=1$.

iii) $S$ has a Galois-invariant double-six. Over the quadratic extension $l / k$, splitting the double-six, $S_{l}$ is isomorphic to $\mathbb{P}^{2}$, blown up in a $k$-rational point, an orbit of size two, and an orbit of size three. Then, $\alpha(S)=1$.

iv) $S$ is isomorphic to $\mathbb{P}^{2}$, blown up in an orbit of size two and an orbit of size four. Then $\alpha(S)=\frac{5}{6}$.

v) $S$ is isomorphic to $\mathbb{P}^{2}$, blown up in a $k$-rational point and an orbit of size five. Then, $\alpha(S)=\frac{17}{24}$.

IV) $\operatorname{rk} \operatorname{Pic}(S)=4$. Then there are three cases.

i) $S$ is isomorphic to $\mathbb{P}^{2}$, blown up in two $k$-rational points and an orbit of size four. Then $\alpha(S)=\frac{5}{18}$.

ii) $S$ is isomorphic to $\mathbb{P}^{2}$, blown up in three orbits of size two. Then $\alpha(S)=\frac{7}{18}$.

iii) $S$ is isomorphic to $\mathbb{P}^{2}$, blown up in a k-rational point, an orbit of size two, and an orbit of size three. Then $\alpha(S)=\frac{3}{8}$. 
$\mathrm{V}) \operatorname{rk} \operatorname{Pic}(S)=5$. Then there are two cases.

i) $S$ is isomorphic to $\mathbb{P}^{2}$, blown up in two $k$-rational points and two orbits of size two. Then $\alpha(S)=\frac{1}{8}$.

ii) $S$ is isomorphic to $\mathbb{P}^{2}$, blown up in three $k$-rational points and an orbit of size three. Then $\alpha(S)=\frac{5}{48}$.

VI) $\operatorname{rk} \operatorname{Pic}(S)=6$. Then $\alpha(S)=\frac{1}{30}$.

VII) $\operatorname{rk} \operatorname{Pic}(S)=7$. Then $\alpha(S)=\frac{1}{120}$.

According to Theorem 4.2 there are 17 conjugacy classes of $\varrho$-maximal subgroups, leading to 14 distinct values of $\alpha(S)$. The 350 conjugacy classes of subgroups and the 91 orbit structures are distributed among them as shown in Table 3 , In case III.i, $(\mathbb{Z} / 2 \mathbb{Z})^{3}$ means the sum zero subspace in $(\mathbb{Z} / 2 \mathbb{Z})^{4}$, acted upon by $\mathrm{S}_{4}$ in the obvious manner.

TABLE 3. Smooth cubic surfaces, the $17 \varrho$-maximal cases

\begin{tabular}{|c|c|c|c|c|c|}
\hline Case & $\alpha$ & $\begin{array}{c}\text { \#conjugacy } \\
\text { classes }\end{array}$ & Q-maximal & $\begin{array}{c}\text { \#orbit } \\
\text { structures }\end{array}$ & maximal \\
\hline $\mathrm{I}$ & 1 & 137 & $W\left(E_{6}\right)$ & 22 & {$[27]$} \\
\hline II.i & 1 & 98 & $W\left(D_{5}\right)$ & 22 & {$[1,10,16]$} \\
\hline II.ii & $\frac{4}{3}$ & 16 & $\mathrm{~S}_{6}$ & 5 & {$[6,6,15]$} \\
\hline II.iii & 2 & 11 & $\mathrm{~S}_{3} \times \mathrm{S}_{3} \times \mathbb{Z} / 2 \mathbb{Z}$ & 3 & {$[3,3,6,6,9]$} \\
\hline II.iv & $\frac{3}{2}$ & 8 & $\mathrm{~S}_{5} \times \mathbb{Z} / 2 \mathbb{Z}$ & 2 & {$[2,5,10,10]$} \\
\hline III.i & $\frac{1}{2}$ & 33 & $\mathrm{~S}_{4} \rtimes(\mathbb{Z} / 2 \mathbb{Z})^{3}$ & 13 & {$[1,1,1,8,8,8]$} \\
\hline III.ii & 1 & 6 & $\mathrm{~S}_{3} \times \mathrm{S}_{3}$ & 3 & {$[3,3,3,3,3,3,9]$} \\
\hline III.iii & 1 & 7 & $\mathrm{~S}_{3} \times \mathrm{S}_{2} \times \mathbb{Z} / 2 \mathbb{Z}$ & 2 & {$[1,2,2,3,3,4,6,6]$} \\
\hline III.iv & $\frac{5}{6}$ & 11 & $\mathrm{~S}_{4} \times \mathrm{S}_{2}$ & 5 & {$[1,2,2,4,4,6,8]$} \\
\hline III.v & $\frac{17}{24}$ & 5 & $\mathrm{~S}_{5}$ & 2 & {$[1,1,5,5,5,10]$} \\
\hline IV.i & $\frac{5}{18}$ & 5 & $\mathrm{~S}_{4}$ & 3 & {$[1,1,1,1,1,4,4,4,4,6]$} \\
\hline IV.ii & $\frac{7}{18}$ & 4 & $\mathrm{~S}_{2} \times \mathrm{S}_{2} \times \mathrm{S}_{2}$ & 3 & {$[1,1,1,2,2,2,2,2,2,4,4,4]$} \\
\hline IV.iii & $\frac{3}{8}$ & 3 & $\mathrm{~S}_{3} \times \mathrm{S}_{2}$ & 1 & {$[1,1,1,2,2,2,3,3,3,3,6]$} \\
\hline V.i & $\frac{1}{8}$ & 2 & $\mathrm{~S}_{2} \times \mathrm{S}_{2}$ & 2 & {$[1,1,1,1,1,1,1,2,2, \ldots, 2,4]$} \\
\hline V.ii & $\frac{5}{48}$ & 2 & $\mathrm{~S}_{3}$ & 1 & {$[1,1, \ldots, 1,3,3,3,3,3,3]$} \\
\hline VI & $\frac{1}{30}$ & 1 & $\mathrm{~S}_{2}$ & 1 & {$[1,1, \ldots, 1,2,2,2,2,2,2]$} \\
\hline VII & $\frac{1}{120}$ & 1 & 0 & 1 & {$[1,1, \ldots, 1]$} \\
\hline$\sum$ & & 350 & & 91 & \\
\hline
\end{tabular}

Remarks 4.3. a) Some of the cases allow equivalent characterizations. For example, IV.i contains the cubic surfaces of Picard rank 4 having five rational lines that form two triangles with a line in common. 
b) Observe that $\operatorname{rk} \operatorname{Pic}(S) \geq 4$ implies that $S$ has a Galois-invariant sixer, i.e., $S$ is then isomorphic over $k$ to the blow-up of $\mathbb{P}^{2}$ in some Galois-invariant set of size six.

c) Recall that gap produces a list, giving the 350 conjugacy classes of subgroups of $W\left(E_{6}\right)$ in a definite numbering. Associated to each number, we have a value $\alpha(S)$. Unfortunately, this list is far too long to be reproduced here. But let us restrict considerations to $\operatorname{rk} \operatorname{Pic}(S) \geq 2$ and one group per orbit structure, the maximal one. Then the situation may be visualized by the inclusion graphs in Figure 1
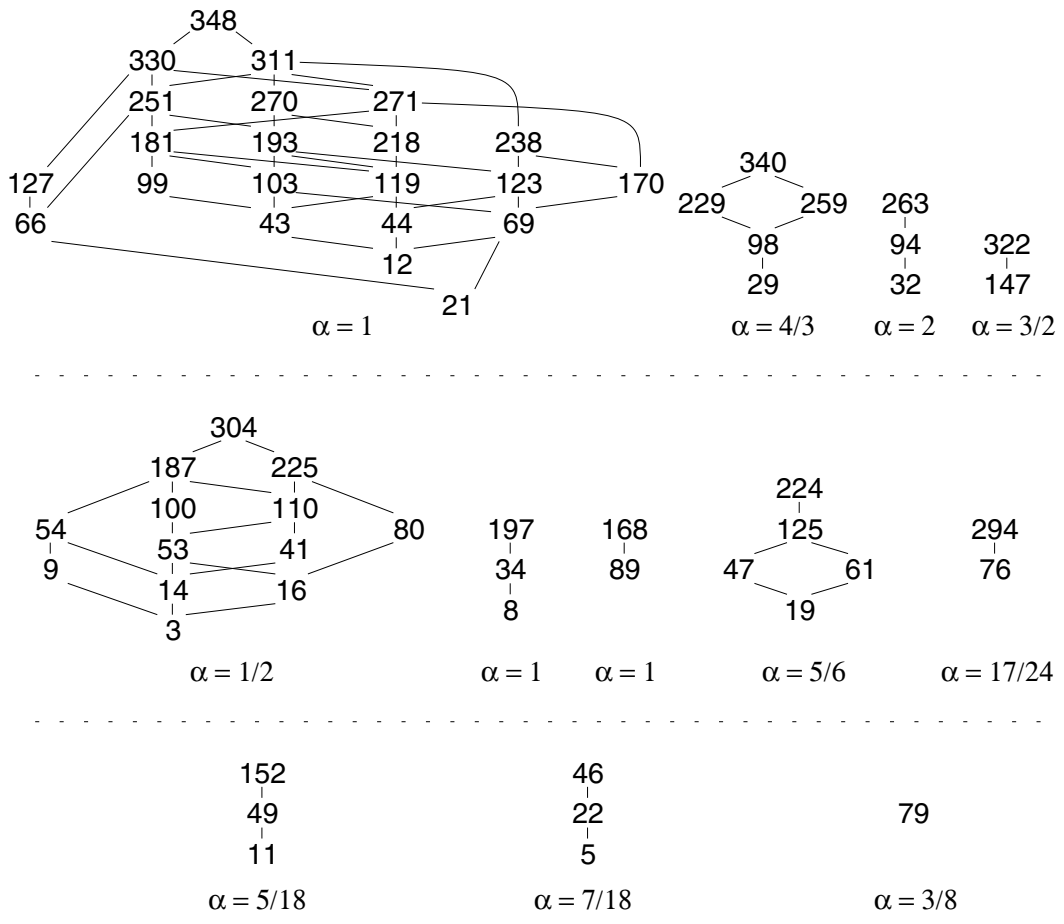

Figure 1. Conjugacy classes of subgroups with Picard ranks 2, 3, and 4 , only one per orbit structure, numbered as in gap 4.4.12.

The four conjugacy classes with Picard rank 5 are 4 and 10, leading to $\alpha=\frac{1}{8}$, as well as 7 and 24, leading to $\alpha=\frac{5}{48}$. Here, 7 and 24 have the same orbit structure. Finally, number 2 is the only with Picard rank 6 and number 1 the only with Picard rank 7.

Example 4.4. Let us take a closer look at case III.v. Here, $S$ is obtained by blowing up $\mathbb{P}^{2}$ in a rational point and an orbit of size five. The Galois group must permute the five points transitively. Therefore, the maximal possible Galois group is isomorphic to $\mathrm{S}_{5}$. It has gap number 294 .

Further, $\mathrm{S}_{5}$ has exactly five conjugacy classes of transitive subgroups. This explains the number 5 in the third column of Table 3 . Among the transitive subgroups, there are the cyclic group of order five and the dihedral group of order 10. For these, from the explicit description of the 27 lines [14, Theorem V.4.9], one easily deduces 
that the finer orbit structure $[1,1,5,5,5,5,5]$ occurs. In fact, the dihedral group is the maximal subgroup corresponding to this orbit structure. Its gap number is 76 .

4.5. For $d=2$ and $d=1$, Theorem 2.2 reduces the problem to 41 respectively 32 conjugacy classes of $\varrho$-maximal subgroups. It is therefore possible to specify all the values of $\alpha(S)$ in Table 4. The subscripts 2 shall indicate that the corresponding rational number arises in two distinct cases.

TABle 4. Del Pezzo surfaces of degrees 2 and 1, the 32 resp. 41 $\varrho$-maximal cases

\begin{tabular}{|c|l|}
\hline $\begin{array}{c}\text { Picard } \\
\text { rank }\end{array}$ & $\alpha$ \\
\hline \hline 1 & 1 \\
\hline 2 & $1,2_{2}, \frac{7}{3}, 3_{2}, 4$ \\
\hline 3 & $1, \frac{5}{3}, \frac{11}{6}, 2, \frac{9}{4}, \frac{5}{2}, \frac{8}{3}, 3_{2}$ \\
\hline 4 & $\frac{2}{3}, \frac{11}{12}, \frac{11}{9}, \frac{13}{9}, \frac{19}{12}, \frac{5}{3}, 2$ \\
\hline 5 & $\frac{17}{36}, \frac{2}{3}, \frac{13}{18}, 1$ \\
\hline 6 & $\frac{7}{30}, \frac{13}{45}$ \\
\hline 7 & $\frac{1}{10}$ \\
\hline 8 & $\frac{1}{30}$ \\
\hline
\end{tabular}

\begin{tabular}{|c|l|}
\hline $\begin{array}{c}\text { Picard } \\
\text { rank }\end{array}$ & $\alpha$ \\
\hline \hline 1 & 1 \\
\hline 2 & $2,4_{2}, \frac{16}{3}, 6,7,8,10$ \\
\hline 3 & $3,4,6, \frac{77}{12}, \frac{23}{3}, \frac{26}{3}, 9, \frac{32}{3}, 11_{2}, 14$ \\
\hline 4 & $4, \frac{35}{6}, \frac{20}{3}, \frac{31}{4}, \frac{85}{9}, \frac{92}{9}, \frac{31}{3}, 13$ \\
\hline 5 & $4, \frac{355}{72}, \frac{41}{6}, \frac{31}{4}, \frac{103}{12}, \frac{92}{9}$ \\
\hline 6 & $\frac{178}{45}, \frac{16}{3}, \frac{94}{15}$ \\
\hline 7 & $\frac{59}{20}, \frac{18}{5}$ \\
\hline 8 & $\frac{29}{15}$ \\
\hline 9 & 1 \\
\hline
\end{tabular}

Remarks 4.6. a) When verifying Manin's conjecture,

$$
N_{U, H}(B) \sim c_{S, H} B(\log B)^{\varrho(S)-1},
$$

for a del Pezzo surface $S$ of degree $d$ over a number field $k$, the rank $\varrho(S)$ of $\operatorname{Pic}(S)$ must be determined, and this is usually done via the action of $\operatorname{Gal}(\bar{k} / k)$ on the set of $(-1)$-curves. With this information, the factor $\alpha(S)$ of $c_{S, H}$ can be read off our tables as follows.

If $S$ is smooth of degree $d \geq 4$ over $k$, we note that the value of $\alpha(S)$ is uniquely determined by $d, \varrho(S)$ and the question whether $S$ contains at least one line defined over $k$.

For $d=3$, the situation is slightly more complicated. With one exception, $\alpha(S)$ can be read off once one has determined $\varrho(S)$, the number of lines defined over $k$ and the numerical orbit structure (i.e., the number of elements in each $\operatorname{Gal}(\bar{k} / k)$ orbit on the set of $(-1)$-curves). Indeed, an analysis of our data shows that $\alpha(S)$ has the same value as the unique one of the $17 \varrho$-maximal cases in Table 3 with the same $\varrho(S)$, the same number of $(-1)$-curves defined over $k$, and an orbit structure that can be split into the given orbit structure (cf. Theorem 2.2), with the following exception.

Numerically, both orbit structures II.ii $[6,6,15]$ and II.iii $[3,3,6,6,9]$ can be split into $[3,3,3,6,6,6]$. These can be distinguished as follows: We are in the first case if and only if one of our three orbits of size 6 consists of pairwise skew lines. 
For $d=2$ and $d=1$, it seems impossible to give a similar strategy because there are 1071 resp. 13975 different orbit structures on the $(-1)$-curves. Furthermore, given a concrete del Pezzo surface of degree 1 or 2 , it might be a delicate problem to determine the corresponding $\varrho$-maximal subgroup as in 2.3 .

b) Several of the values have been known before. For $\operatorname{rk} \operatorname{Pic}(S)=1$, the value $\alpha(S)=1$ is almost immediate from E. Peyre's definition. For split del Pezzo surfaces, our calculations confirm the values from [7, Theorem 4].

In degree 4, case V confirms the value in [3, Section 10]. In degree 3, II.i and II.iv are shown in [15, Remarks VI.5.9]. II.ii is [15, Example 5.6]. Further, particular cases of II.iii, III.iii, and IV.ii appear in [18, Proposition 5.1].

\section{REFERENCES}

[1] V.V. Batyrev and Yu. Tschinkel, Rational points of bounded height on compactifications of anisotropic tori, International Mathematics Research Notices (1995), 591-635. MR.1369408 (97a:14021)

[2] W. Bosma, J. J. Cannon, C. Fieker, and A. Steel, (eds.): Handbook of Magma functions, Edition 2.16 (2010)

[3] R. de la Bretèche and T. D. Browning, Manin's conjecture for quartic del Pezzo surfaces with a conic fibration, Duke Math. J. 160 (2011), no. 1, 1-69, DOI 10.1215/00127094-1443466. MR2838351(2012k:11038)

[4] T. D. Browning, An overview of Manin's conjecture for del Pezzo surfaces, Analytic number theory, Clay Math. Proc., vol. 7, Amer. Math. Soc., Providence, RI, 2007, pp. 39-55. MR2362193 (2008j:14041)

[5] John Cannon and Derek F. Holt, Computing maximal subgroups of finite groups, J. Symbolic Comput. 37 (2004), no. 5, 589-609, DOI 10.1016/j.jsc.2003.08.002. MR2094616|(2005i:20047)

[6] Jean-Louis Colliot-Thélène and Jean-Jacques Sansuc, La descente sur les variétés rationnelles. II, Duke Math. J. 54 (1987), no. 2, 375-492, DOI 10.1215/S0012-7094-87-05420-2 (French). MR899402 (89f:11082)

[7] Ulrich Derenthal, On a constant arising in Manin's conjecture for del Pezzo surfaces, Math. Res. Lett. 14 (2007), no. 3, 481-489. MR2318651 (2008i:14053)

[8] Ulrich Derenthal, Michael Joyce, and Zachariah Teitler, The nef cone volume of generalized del Pezzo surfaces, Algebra Number Theory 2 (2008), no. 2, 157-182, DOI 10.2140/ant.2008.2.157. MR2377367(2009b:14069)

[9] Andreas-Stephan Elsenhans and Jörg Jahnel, Cubic surfaces with a Galois invariant double-six, Cent. Eur. J. Math. 8 (2010), no. 4, 646-661, DOI 10.2478/s11533-010-0036-1. MR2671217(2011e:14045)

[10] Andreas-Stephan Elsenhans and Jörg Jahnel, On the Brauer-Manin obstruction for cubic surfaces, J. Comb. Number Theory 2 (2010), no. 2, 107-128. MR2907786

[11] Jens Franke, Yuri I. Manin, and Yuri Tschinkel, Rational points of bounded height on Fano varieties, Invent. Math. 95 (1989), no. 2, 421-435, DOI 10.1007/BF01393904. MR.974910 (89m:11060)

[12] The GAP Group: GAP - Groups, Algorithms, and Programming, Version 4.4.12, 2008, http://www.gap-system.org

[13] Ewgenij Gawrilow and Michael Joswig, polymake: a framework for analyzing convex polytopes, Polytopes - combinatorics and computation (Oberwolfach, 1997), DMV Sem., vol. 29, Birkhäuser, Basel, 2000, pp. 43-73. MR1785292 (2001f:52033)

[14] Robin Hartshorne, Algebraic geometry, Springer-Verlag, New York, 1977. Graduate Texts in Mathematics, No. 52. MR.0463157(57 \#3116)

[15] J. Jahnel, Brauer groups, Tamagawa measures, and rational points on algebraic varieties, Habilitation thesis, Göttingen, 2008.

[16] Yu. I. Manin, Cubic forms: algebra, geometry, arithmetic, North-Holland Publishing Co., Amsterdam, 1974. Translated from the Russian by M. Hazewinkel; North-Holland Mathematical Library, Vol. 4. MR0460349 (57 \#343) 
[17] Emmanuel Peyre, Hauteurs et mesures de Tamagawa sur les variétés de Fano, Duke Math. J. 79 (1995), no. 1, 101-218, DOI 10.1215/S0012-7094-95-07904-6 (French). MR1340296 (96h:11062)

[18] Emmanuel Peyre and Yuri Tschinkel, Tamagawa numbers of diagonal cubic surfaces of higher rank, Rational points on algebraic varieties, Progr. Math., vol. 199, Birkhäuser, Basel, 2001, pp. 275-305. MR1875177(2003a:11076)

Mathematisches Institut, Ludwig-Maximilians-Universität München, Theresienstr. 39, D-80333 MÜNCHEN, GERMANY

E-mail address: ulrich.derenthal@mathematik.uni-muenchen.de

School of Mathematics and Statistics F07, University of Sydney, NSW 2006, Sydney, Australia

E-mail address: stephan@maths.usyd.edu.au

Département Mathematik, Universität Siegen, Walter-Flex-Str. 3, D-57068 Siegen, GERMANY

E-mail address: jahnel@mathematik.uni-siegen.de 